De Rijcke, S., Tielens, P., Rawn, B., Van Hertem, D., Driesen, J. (2014). Trading Energy Yield for Frequency Regulation: Optimal Control of Kinetic Energy in Wind Farms. IEEE Transactions on Power Systems.

This is the author's version of an article that has been accepted for publication in IEEE Transactions on power delivery. Changes are made to this version by the publisher prior to publication.

(C)2014 IEEE. Personal use of this material is permitted. Permission from IEEE must be obtained for all other users, including reprinting/ republishing this material for advertising or promotional purposes, creating new collective works for resale or redistribution to servers or lists, or reuse of any copyrighted components of this work in other works. 


\title{
Trading Energy Yield for Frequency Regulation: Optimal Control of Kinetic Energy in Wind Farms
}

\author{
Simon De Rijcke, Graduate Student Member, IEEE, Pieter Tielens, Graduate Student Member, IEEE,
} Barry Rawn, Member, IEEE, Dirk Van Hertem, Senior Member, IEEE, and Johan Driesen, Senior Member, IEEE

\begin{abstract}
The burden on conventional units to regulate the system frequency increases if they are replaced due to wind farms. This paper explores up to which time scales the rotating kinetic energy in wind turbines can smooth frequency variations and assist with the regulation task. To this end, a comparison is made between a standard wind turbine controller and optimal control of wind turbines, respectively derived from causal timedomain simulations and an optimization algorithm that allows predicting. The latter algorithm is used to give a benchmark for the smoothing potential, shown by plotting the Pareto efficiency of the normalized standard deviation of frequency variability versus a normalized measure of the energy yield. Results indicate that smoothing comes with an energy loss that is determined by the energy content of power imbalances. It is shown that a wind share of $20 \%$, within the instantaneous generation mix, can absorb frequency variations on timescales up to $100 \mathrm{sec}$ while the energy loss is limited to only $2 \%$. A higher share of wind power aggravates frequency variability. Nevertheless, in such circumstances the potential of rotating kinetic energy in wind farms increases.
\end{abstract}

Index Terms-Smooth frequency variability, optimization, frequency regulation, wind farm control, maximum energy yield

LIST OF SYMBOLS

\begin{tabular}{ll}
\hline Symbol & Description \\
\hline$A$ & Rotor surface area \\
$C_{p}$ & Aerodynamic coefficient of performance \\
$E$ & Energy yield \\
$E_{k}$ & Rotating kinetic energy \\
$H$ & Inertia constant \\
$I$ & Moment of inertia \\
$J$ & Objective function \\
$\mathscr{J}$ & Cost functional \\
$K$ & Proportional gain \\
$\Delta L$ & Magnitude of load changes \\
$P$ & Active power \\
$P_{a}$ & Aerodynamic power \\
$P_{e}$ & Electric power of one turbine \\
$P_{f}$ & Electric power of wind farm \\
$P_{g}$ & Electric power of thermal units \\
$P_{L}$ & Electric power of load \\
$P_{r e s}$ & Upward power reserve \\
$R$ & Rotor radius \\
$R_{g}$ & Droop setting of thermal units \\
$R_{w}$ & Droop setting of wind turbines \\
$s$ & Laplace variable \\
\end{tabular}

S. De Rijcke, P. Tielens, B. Rawn, D. Van Hertem and J. Driesen are with KU Leuven, Department ESAT, research group Electa Heverlee, 3001, Belgium. E-mail: simon.derijcke@esat.kuleuven.be, pieter.tielens@esat.kuleuven.be.

$\mathrm{KU}$ Leuven ESAT/Electa is a founding member of Energyville.
Time

Time period

Aerodynamic torque

Electric torque

Optimization vector of electric torques

Valve position of governor

Rotating kinetic energy exchange

Pareto weight

Pitch angle

Relative kinetic energy exchange

Tip-speed ratio

Share of wind power in generation mix

Air density

Time lag/constant

Governor time constant

Turbine time constant

Rotor speed (referred to low speed shaft)

Optimization vector of rotor speeds

Power system frequency

\begin{tabular}{ll} 
Superscript & Description \\
\hline$*$ & Reference value
\end{tabular}

\section{INTRODUCTION}

Q YSTEM operators are responsible for maintaining the balance between generation and load, which requires a continuous regulation effort due to constant load and generation fluctuations. Load fluctuations range from minuteto-minute variability, due to random turning on and off of millions of devices, to longer-term variability such as daily, seasonal and annual patterns that are predictable to a large extent [1]. Variability introduced by generators is due to the unit commitment process following the load evolutions. Additionally, variability is increased by deviations from nomination schedules and the variable nature of Renewable Energy 
Sources (RES) such as wind and solar.

The restructuring of the energy market and changing generation sources are challenging aspects that force a closer look at the mechanisms needed to meet this balance [2]. First, these market changes reinforced energy trading significantly: for instance cross-border schedules are more intensively used for trading. They are bound to standardized time intervals, at which balance responsible parties have to abide by energy nomination schedules. At times of high power ramps, e.g. in the morning and evening, mismatches tend to arise between nominated energy blocks at fixed time periods, and demand [3]. These mismatches provoke large frequency swings within a time window centered around standardized cross-border time intervals, requiring power regulation mechanisms. Secondly, the increasing penetration of converter coupled sources entails a decommitment of conventional, mostly thermal, units equipped with synchronous generators. Thermal time constants and controls of such units absorb to a large extent minute-tominute frequency variations, for instance resulting from wind power fluctuations [4]. The displacement of thermal units with a critical role in the system reduces this absorption effect and requires sufficient rotational energy and reserves from remaining units. Thirdly, RES make the frequency regulation task more difficult, as wind and solar are variable sources of energy. Wind fluctuations for instance range from intra-minute to even annual time scales [5]. This can result in very steep ramps when wind farms are gathered in a small geographical area, with operational difficulties as a consequence.

Although wind is mostly perceived as merely a contributor to frequency variability, it can help with the task of regulation. The variable speed capability of such turbines allows a much greater exploitation of rotational energy taking rotor speed and converter limits into account [6]. Over large geographical areas, the aggregate capability of a wind farm fleet can be relevant [7], [8].

Contrary to most wind turbine controllers that have been developed primarily to mitigate severe frequency dips following large power imbalances [9]-[12], the focus is on the aspect of frequency regulation. Note that we distinguish frequency control during contingencies and normal operation: the former refers to frequency response control while the latter indicates frequency regulation [2]. They are both incorporated into the Frequency Containment Reserves (FCR) - also known as primary reserves - as introduced by the European Network for Transmission System Operators for electricity (ENTSOE) [13]. To release the burden of regulation on conventional units, we introduce an optimal control problem that examines which system frequency variations can be mitigated by the use of kinetic energy in wind turbines. The resulting power references minimize the energy loss and provide the highest achievable smoothing of the system frequency.

This paper makes several new contributions beyond existing literature in this area. The prospect of smoothing variations of the combined system of wind farm and bulk power system is considered, rather than that of smoothing variations from the farm alone [14]. The limits on energy exchange capability of a whole farm, rather than a single turbine [15] are studied. The efficient use of kinetic energy in the rotating parts is investigated, while often energy storage systems are proposed to smooth wind or frequency variations within the range of seconds to minutes [16]. Finally, the optimization framework used includes the possibility of benefits through coordination between turbines in a farm, rather than issuing the same command to all turbines.

Some controllers do focus on the mitigation of wind power variability in favor of frequency stability [17], [18], but the exploration of time-scales up to which wind farms are able to smooth system frequency variations remains largely unexplored. The smoothing capability of wind farms is investigated in this paper for two instantaneous penetration levels of wind power, respectively $20 \%$ and $90 \%$. Such high levels require an increasing contribution from wind power to support the frequency in terms of regulation.

The paper is structured as follows. First, Section II highlights the approach and methodology used. Thereafter, Section III introduces the power system model and optimization problem. The results in Sections IV and V show the ability to smooth frequency variations starting from sinusoidal and representative load profiles and discuss the impact of a higher wind penetration on the smoothing potential. Finally, Section VI summarizes the conclusions.

\section{Methodology \& Assumptions}

By means of an optimization problem, the ability of wind farms to smooth system frequency variations is investigated. Hereby, energy loss and frequency variations are minimized. The optimal solution is the best case scenario that cannot be surpassed by any controlled wind turbine and is intended to show the potential of coordination and forecasting. The comparison with time-domain simulations using a standard wind turbine controller - that is designed to mitigate severe frequency swings [9] - is merely to benchmark this potential. The need for such frequency control stems from the loss of control response from regular generators when they are turned off in favor of wind being turned on. This effect is modeled by adjusting the fraction of wind $(\mu=20 \%, 90 \%)$ and conventional $(1-\mu)$ generation. Both the optimization algorithm and time-domain simulations with a standard controller employ the same power system model and result in time-series of control and state variables. They only differ in their strategy to control the wind turbines, either optimally or using a standard controller.

A simplified power system model is used that consists of an equivalent thermal unit and an equivalent wind farm, respectively aggregating all thermal and wind units in the generation mix (cfr. III-A). The power system is modeled by a swing equation with lumped inertia and the equivalent thermal unit is represented by a governor. By neglecting load dynamics, a worst case scenario is simulated. Also area generation control (AGC) is omitted as we are not interested in slower control actions and exchange schedules between control areas. The equivalent wind farm collects all wind units in the generation mix into two groups. In each group, all turbines are modeled by an aggregated variable-speed wind turbine model facing respectively a constant wind speed of 
8 and $10 \mathrm{~m} / \mathrm{s}$. Such modeling has several consequences. Firstly, a constant wind speed neglects wind power variability. Our focus on the ability to smooth system frequency variations, not wind power variations, motivates this choice. Secondly, only the variable-speed operating region (region II) is considered, thus not imposing rotor speed limits. This choice allows to freely steer the kinetic energy content and to easily compare with the standard controller. Unacceptable rotor speeds that exceed the limits are tracked and if necessary their relevance is mentioned. Further research requires a strict limitation on the rotor speeds, incorporating other operating regions and lowering the smoothing potential. Thirdly, division of the wind farm over two groups allows to include possible coordination among both turbines facing a different wind speed.

\section{ModELING}

First, §III-A elaborates on the power system model and introduces a standard wind turbine controller for frequency support. Thereafter, $\S$ III-B constructs the optimization formulation, using the same power system model, to retrieve optimal control actions that smooth power system frequencies at the lowest energy loss. Finally, §III-C clarifies the computational set-up.

\section{A. Time-domain modeling}

A simplified power system model is created by combining the system's swing equation and governing actions of all units in operation [12], illustrated in Figure 1a. For load-frequency studies, the swing equation that represents the response of the power system frequency to variations in load and generation is expressed in terms of power:

$$
2 H \frac{\mathrm{d} \Delta \omega_{s}}{\mathrm{~d} t}=(1-\mu) \Delta P_{g}+\mu \Delta P_{f}-\Delta P_{L}
$$

where $H$ represents the equivalent system inertia and $\mu$ the share of wind generation [19]. Furthermore, $\Delta \omega_{s}, \Delta P_{g}$, $\Delta P_{f}$ and $\Delta P_{L}$ give the linearized deviation of respectively the system frequency $\omega_{s}$, conventional generation $P_{g}$, wind farm generation $P_{f}$ and the system load $P_{L}$ with respect to their reference. These references are set to the initial values $\left(P_{g, 0}=0, P_{L, 0}=0, \omega_{s, 0}=50 \mathrm{~Hz}\right)$, neglecting non-linear effects. $P_{f, 0}$ is set to the maximum power for a given wind speed $\left(P_{m p p t}\right)$, subtracted by the reserve power $\left(P_{r e s}\right)$, used for delivering frequency support (cfr. $\S$ III-A2).

1) Thermal unit model: Conventional generation $\Delta P_{g}$ is aggregated into one governing action from a thermal unit, determined by a droop action $R_{g}$. This action is subject to two time constants $\tau_{g}$ and $\tau_{t}$, representing respectively the governor and turbine. The model for this generator, illustrated in Figure 1b, comprises two differential equations:

$$
\begin{aligned}
\tau_{g} \frac{\mathrm{d} \Delta y}{\mathrm{~d} t} & =\frac{-\Delta \omega_{s}}{R_{g}}-\Delta y, \\
\tau_{t} \frac{\mathrm{d} \Delta P_{g}}{\mathrm{~d} t} & =\Delta y-\Delta P_{g}
\end{aligned}
$$

where $\Delta y$ is the valve position.

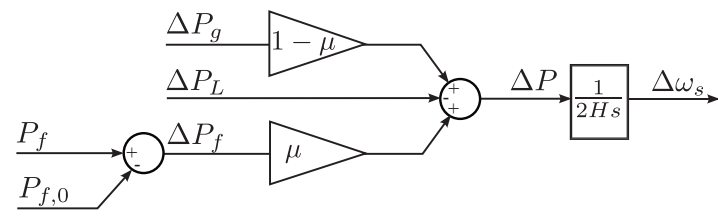

(a)

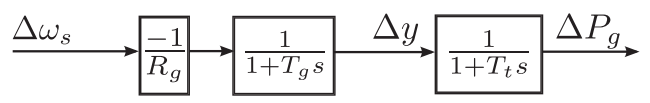

(b)

Fig. 1: Power system model with wind and conventional generation (a) and the speed governor of the thermal unit (b)

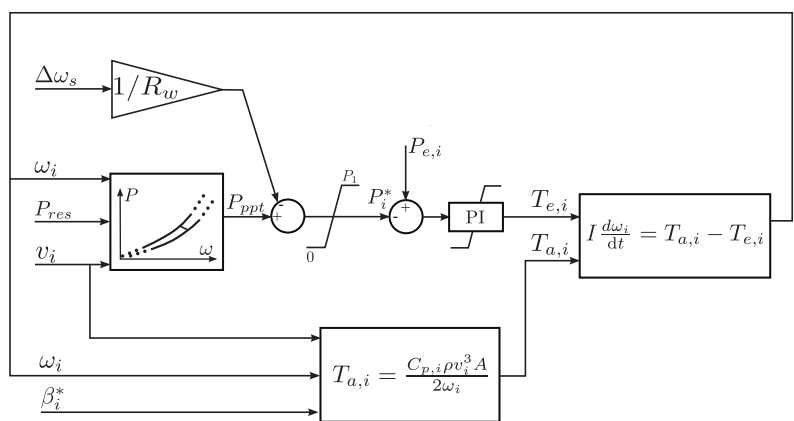

Fig. 2: Wind turbine model. All variables are referred to the low speed shaft.

2) Wind turbine model: Different control strategies exist to regulate variable speed wind turbines [20], [21]. Nevertheless, all strategies use a wind turbine controller that consists of two cascaded control levels. The outer-loop controller regulates either the rotor speed or the power and creates a reference current signal for the converter. In this work, one state-of-theart power control strategy is preferred for the converter [9], further referred to as standard control. For such a strategy, the speed is normally limited by a pitch controller, however not modeled as we only consider operating region II. The innerloop controller regulates the current to a reference value. This lower control level and the converter dynamics are orders of magnitude faster than dynamic phenomena included in this study, so the current is assumed equal to the reference. This assumption allows flux and torque to be viewed as control inputs. Moreover, also the stator and rotor flux transients are much faster which allows us to use a first order model for each generator, referred to the low speed shaft,

$$
I \frac{\mathrm{d} \omega_{i}}{\mathrm{~d} t}=T_{a, i}-T_{e, i}
$$

where $I$ is the moment of inertia from turbine and generator, $T_{e, i}$ is the electric torque, $T_{a, i}$ the aerodynamic torque and $\omega_{i}$ the rotational speed of turbine $i=1 \ldots N$. The electric torque $T_{e, i}$ follows directly from the power controller (cfr. Figure 2). The aerodynamic torque $T_{a, i}$ on the other hand can be expressed starting from the aerodynamic power $P_{a, i}$ :

$$
P_{a, i} \equiv \frac{1}{2} C_{p, i} \rho A v_{i}^{3}=T_{a, i} \omega_{i} \Rightarrow T_{a, i}=\frac{C_{p, i} \rho A v_{i}^{3}}{2 \omega_{i}}
$$


with $A=\pi R^{2}$ the rotor area, $R$ the rotor radius, $\rho$ the air density, $C_{p, i}$ the aerodynamic coefficient of performance and $v_{i}$ the wind velocity. An empirical relation for $C_{p}$ is used that corresponds to [22], [23]:

$$
\begin{aligned}
C_{p}\left(\lambda_{i}, \beta_{i}\right) & =c_{1}\left(c_{2} \gamma_{i}+c_{3} \beta_{i}+c_{4}\right) e^{c_{5} \gamma_{i}} \\
\text { with } \gamma_{i} & =\frac{1}{\lambda_{i}+c_{6} \beta_{i}}+\frac{c_{7}}{\beta_{i}^{3}+1}
\end{aligned}
$$

and where $\lambda_{i}=R \omega_{i} / v_{i}$ is the tip-speed ratio. Further, $c_{1}=$ $0.22, c_{2}=116, c_{3}=-0.4, c_{4}=-5, c_{5}=-12.5, c_{6}=0.08$ and $c_{7}=-0.035$.

The power set-point tracking of $P_{p p t}$ in the wind turbine controller, depicted in Figure 2, is based on a linear slope between two power-speed curves [9]. Without a power reserve requirement $P_{\text {res }}=0$, the turbine tracks maximum efficiency, also called maximum power-point tracking (MPPT). Consequently, turbine control is based on the MPPT curve in Figure 3 and would result in a setpoint $P_{m p p t}\left(v_{i}\right)$, dependent on the wind speed $v_{i}$. A deloaded curve $\left(P_{d e l}\right)$ is constructed by a power reserve $P_{\text {res }}$ request above zero, thus

$$
P_{\text {del }}\left(v_{i}\right)=P_{\text {mppt }}\left(v_{i}\right)-P_{\text {res }} .
$$

However, the deloaded curve in Figure 3 is not used as a control curve but serves as input for the power set-point tracking, calculated as

$$
\begin{aligned}
P_{p p t}\left(\omega_{i}, v_{i}\right)=\frac{P_{m p p t}\left(v_{i}\right)-P_{d e l}\left(v_{i}\right)}{\omega_{m p p t}\left(v_{i}\right)-\omega_{\text {del }}\left(v_{i}\right)}\left(\omega_{i}\right. & \left.-\omega_{d e l}\left(v_{i}\right)\right) \\
& +P_{d e l}\left(v_{i}\right),
\end{aligned}
$$

where the corresponding rotor speeds $\omega_{m p p t}\left(v_{i}\right)$ and $\omega_{\text {del }}\left(v_{i}\right)$ are obtained from respectively the MPPT and deloaded powerspeed curves. By applying this control, additional kinetic energy will be released during frequency support since the operating point of the turbine $\left(P_{p p t}\left(\omega_{i}, v_{i}\right)\right)$ will move along the linear curve (cfr. Eq. (8)) from $P_{\text {del }}\left(v_{i}\right)$ towards $P_{m p p t}\left(v_{i}\right)$. The resulting set-point $P_{p p t}\left(\omega_{i}, v_{i}\right)$ is then increased with the primary response $-\Delta \omega_{s} / R_{w}$ to correct for frequency deviations. A reference power $P_{i}^{*}$ is obtained, which is limited by the maximum available power at a certain wind speed $\left(P_{m p p t}\right)$ in order to ensure a stable frequency support during longer time periods. Otherwise, if the power set point exceeds the available power in case of a severe frequency dip, the kinetic energy in the wind turbine gets depleted and the rotor speed drops to zero.

The absolute farm power $P_{f}$ is defined by the electric power of each turbine $P_{e, i}$ and is expressed as

$$
P_{f}=\sum_{i=1}^{N} P_{e, i}=\sum_{i=1}^{N} T_{e, i} \omega_{i},
$$

\section{B. Formulation of optimization problem}

Objectives: The optimal control of a wind farm is considered by means of the electrical torques $\boldsymbol{T}_{\boldsymbol{e}}(t)$ of all turbines in the farm. Here, $\boldsymbol{T}_{\boldsymbol{e}}$ is a vector containing the torques $T_{e, i}(t)$ of individual turbines, with $i=1 \ldots N$ turbines. Pitch angles $\beta_{i}(t)$ of individual turbines, combined in vector $\boldsymbol{\beta}(t)$, are set to a fixed angle corresponding to maximum efficiency. By doing

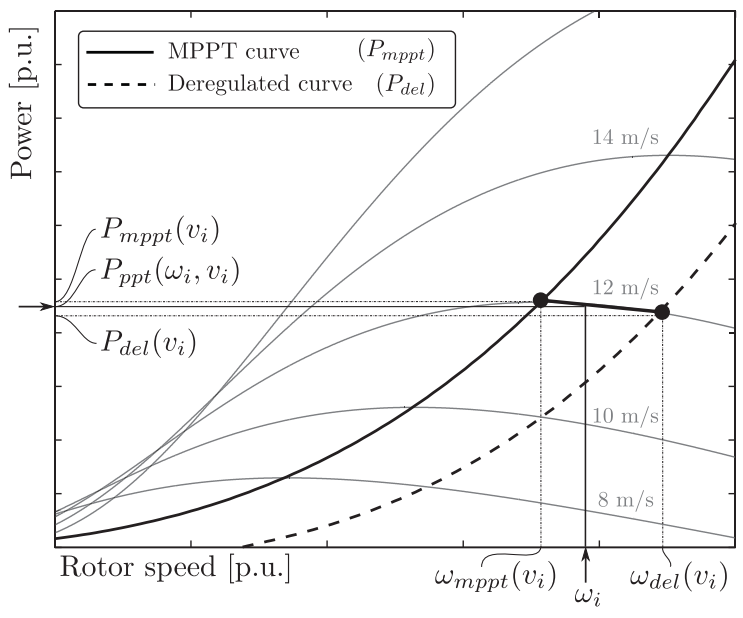

Fig. 3: Schematic representation of power set-point tracking $P_{p p t}$.

so, a comparison with the earlier introduced standard controller is achieved, for which pitch control is also disabled. The torques $T_{e, i}(t)$ dynamically depend on time and are optimized in a time window $0<t<T$. By using these control variables, we aim at two competing objectives: i.e. maximization of total energy extraction $\mathscr{J}_{1}$, and minimization of system frequency fluctuations $\mathscr{J}_{2}$,

$$
\begin{array}{r}
\mathscr{J}_{1}\left(\boldsymbol{\omega}, \boldsymbol{T}_{\boldsymbol{e}}, T\right)=\int_{0}^{T}-P_{f}\left(\boldsymbol{\omega}(t), \boldsymbol{T}_{\boldsymbol{e}}(t)\right) \mathrm{d} t \\
=\int_{0}^{T}-\sum_{i=1}^{N} T_{e, i}(t) \omega_{i}(t) \mathrm{d} t \\
\mathscr{J}_{2}\left(\Delta \omega_{s}, T\right)=\int_{0}^{T} \Delta \omega_{s}(t)^{2} \mathrm{~d} t
\end{array}
$$

with $\boldsymbol{\omega}(t)$ the vector with rotational speeds of the different turbines and $\Delta \omega_{s}(t)$ the system frequency deviations. Following standard conventions in optimization, the signs of $\mathscr{J}_{1}$ and $\mathscr{J}_{2}$ are chosen in a way the optimization is formulated as a minimization problem. The first objective $\mathscr{J}_{1}$, maximizes the energy yield of the entire wind farm by optimizing torques $T_{e, i}(t)$, simultaneously coordinating all $N$ turbines' operating points. The second objective $\mathscr{J}_{2}$ minimizes system frequency deviations, by penalizing frequency deviations at every time instance.

The overall optimization problem is formulated based on a weighted sum of both (competing) cost functionals, i.e.

$$
\begin{aligned}
\mathscr{J} & =\alpha \mathscr{J}_{1}+(1-\alpha) \mathscr{J}_{2} \\
& =\int_{0}^{T}\left[-\alpha \sum_{i=1}^{N} T_{e, i}(t) \omega_{i}(t)+(1-\alpha) \Delta \omega_{s}(t)^{2}\right] \mathrm{d} t
\end{aligned}
$$

where we vary $\alpha \in[0,1]$ in order to construct Pareto fronts (cfr. $\S \mathrm{IV}$ ), and explore different trade-offs between $\mathscr{J}_{1}$ and $\mathscr{J}_{2}$. 
Constraints: The wind turbine and grid model, introduced in $§ I I I-A$, set the constraints. Contrary to the time-domain where the control set-up in Figure 2 determines the controls $T_{e, i}$, the optimization algorithm is free to optimize these controls.

The farm power $P_{f}$ that appears in $\mathscr{J}_{1}$ depends on the controls $\boldsymbol{T}_{\boldsymbol{e}}$, but further also on the rotational speeds $\boldsymbol{\omega}$ of the turbines. The latter is a state variable that is constrained by Newton's second law for rotation, given by Eq. (4). The system frequency $\Delta \omega_{s}(t)$, being a state variable in the optimization set-up, is constrained by the swing equation of the power system, earlier introduced in Eq. (1). Conventional power generation is determined by the governor and turbine, represented by two differential equations (2) and (3). $\Delta P_{g}$ is thus handled as a state variable and is not controllable.

In summary, the optimization problem is

$$
\begin{array}{ll}
\min _{\boldsymbol{T}_{\boldsymbol{e}}} & \int_{0}^{T}\left[-\alpha \sum_{i=1}^{N} T_{e, i}(t) \omega_{i}(t)+(1-\alpha) \Delta \omega_{s}(t)^{2}\right] \mathrm{d} t \\
\text { s.t. } & I \frac{\mathrm{d} \boldsymbol{\omega}}{\mathrm{d} t}=\boldsymbol{T}_{\boldsymbol{a}}\left(\boldsymbol{V}_{\infty}, \boldsymbol{\omega}, \boldsymbol{\beta}\right)-\boldsymbol{T}_{\boldsymbol{e}} \\
& 2 H \frac{\mathrm{d} \Delta \omega_{s}}{\mathrm{~d} t}=(1-\mu) \Delta P_{g}+\mu \Delta P_{f}-\Delta P_{L} \\
& \frac{\mathrm{d} \Delta y}{\mathrm{~d} t}=\frac{-\Delta \omega_{s}}{R_{g} \tau_{g}}-\frac{\Delta y}{\tau_{g}} \\
& \frac{\mathrm{d} \Delta P_{g}}{\mathrm{~d} t}=\frac{1}{\tau_{t}}\left(\Delta y-\Delta P_{g}\right)
\end{array}
$$

Furthermore we do not limit the rotor speeds, neither the rotor torques to allow a transparent comparison between the timedomain (standard controller) and optimization simulations. Nevertheless, all simulation results are screened for unreasonable values of the turbine controls and states.

\section{Computational set-up}

To solve the optimization problem, all equations are first discretized in time. To that end, the state variables $\boldsymbol{\omega}(t)$ are sampled every $0.2 \mathrm{sec}$ and the constraints in Eqs. (13) are discretized using the trapezoid rule according to NewtonCotes. The controls $\boldsymbol{T}_{\boldsymbol{e}}(t)$ are allowed to change every $1.0 \mathrm{sec}$ and remain constant during intermediate time steps of state variables. Given the frequency content of the wind signal $(<0.5 \mathrm{~Hz})$ and the size of turbine inertia, we verified that this discretization gives sufficient accuracy, i.e. further timestep refinement does not lead to noticeably different results. The time-domain simulations with standard controller are performed by a continuous solver, after which the states and controls are respectively sampled every $0.2 \mathrm{sec}$ and $1.0 \mathrm{sec}$. This sampling allows a comparison with the optimization results. The optimization problem stated in Eqs. (13) is solved by a gradient based solver from TOMLAB/KNITRO [24]. This solver offers an iterative conjugate gradient approach for large scale problems. The time-domain model is simulated using MATLAB/Simulink. A simulation time window of $T=800 \mathrm{sec}$ is selected. However, only data within $50<t<650 \mathrm{sec}$ is used to omit boundary effects.

\section{SINUSOIDAL LOAD PROFILES}

In this first case, we analyze the ability of wind turbines to smooth system frequency variations that arise from load variability, being the only source of variations in the chosen power system model. In particular, sinusoïdal load variations are chosen to investigate the energy loss that comes with power smoothing at specified frequencies. Wind turbines are aggregated in two large groups as described in $\S I I$, eliminating wind power variability at power system level. The wind turbines represent a share of $20 \%(\mu=0.2)$ of demand. The load varies with frequency $2 \pi / T_{L}$ :

$$
\Delta P_{L}(t)=\Delta L \cdot \sin \left(2 \pi / T_{L} \cdot t\right)
$$

where the amplitude $\Delta L$ of the load changes is set to 0.0125 , $0.025,0.05$ and 0.10 p.u., and the period $T_{L}$ takes on values of $25,50,100,200$ and 300 seconds. For every combination of $T_{L}$ and $\Delta L$, the cost to smooth these load variations is analyzed, which allows to investigate to which time scale, i.e. in which frequency range, optimal control of kinetic energy in turbines is worthwhile. In the current work Pareto fronts are used to address the trade-off between two competing objectives, i.e. maximum energy yield versus minimum system frequency variations, respectively expressed by

$$
\begin{aligned}
E & =\int_{0}^{T} \sum_{i=1}^{N} T_{e, i}(t) \omega_{i}(t) \mathrm{d} t \\
\sigma & =\sqrt{\frac{1}{T} \int_{0}^{T}\left(\Delta \omega_{s}(t)-\overline{\Delta \omega_{s}}\right)^{2} \mathrm{~d} t}
\end{aligned}
$$

where $E$ is the energy yield from wind turbines and $\sigma$ is the standard deviation of frequency deviations $\Delta \omega_{s}$. These metrics are calculated using a sampling of $1 \mathrm{sec}$ and are normalized to

$$
\begin{aligned}
E_{*} & =\frac{E}{[E]^{\alpha=1}} \\
\sigma_{*} & =\frac{\sigma}{[\sigma]^{\alpha=1}}
\end{aligned}
$$

where $[E]^{\alpha=1}$ and $[\sigma]^{\alpha=1}$ are respectively the energy extraction and standard deviation when only maximizing energy yield. The same normalization is used for results from the time-domain and optimization simulation, because results for $\alpha=1$ are quasi identical. In the remainder of this section, we illustrate the trade-off between $E_{*}$ versus $\sigma_{*}$ for timedomain and optimization simulations. Data of conventional and wind turbine units are given in Appendix I. The base value for parameters in per unit is set to $S_{b}=10 \mathrm{MVA}$.

\section{A. System frequency smoothing: standard control}

First $\alpha=1$, corresponding to maximum energy yield without smoothing frequency fluctuations. This corresponds to $\left(\sigma_{*}\right.$, $\left.E_{*}\right)=(1,1)$ on the Pareto front shown in Figure 4. Thereafter, system frequency variations are minimized by increasing the power reserve $P_{\text {res }}$ stepwise. This action serves the aim to allow an increasing contribution from wind turbines to smooth system frequency variations. As a consequence, energy yield $E_{*}$ lowers proportionally with $P_{\text {res }}$. To make efficient use of 


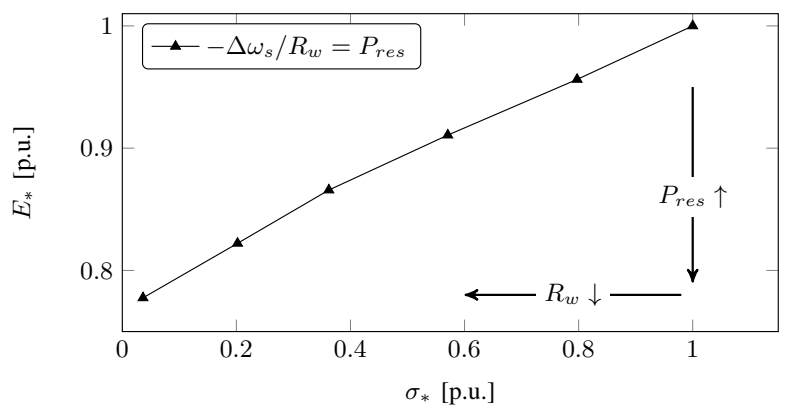

Fig. 4: Efficiency of a standard controller to smooth frequency variability, when subject to a sinusoidal load with $T_{L}=50 \mathrm{sec}$ and $\Delta L=0.05$ p.u.

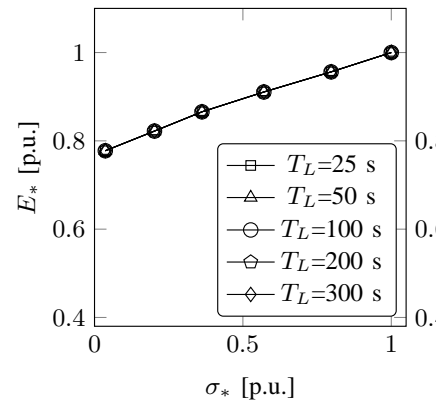

(a)

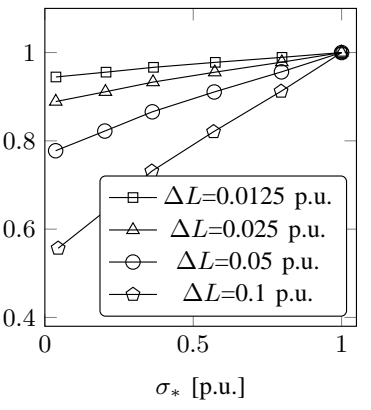

(b)
Fig. 5: Pareto front for a standard controller (a) where the load period $T_{L}$ is varied with $\Delta L=5 \%$, and (b) where the amplitude $\Delta L$ is varied with $T_{L}=50 \mathrm{sec}$.

the available power reserves, a droop action of the wind farm is simulated by lowering droop constant $R_{w}$. Subsequently, grid variability $\sigma_{*}$ is reduced for each $P_{\text {res }}$. Next, an overlay front as shown in Figure 4 is constructed to connect the combinations of $R_{w}$ and $P_{\text {res }}$ for which the frequency response equals the available reserve power, i.e. $-\Delta \omega_{s} / R_{w}=P_{\text {res }}$. This front shows the variability $\sigma_{*}$ for a given energy yield $E_{*}$ and indicates the trade-off for the proposed frequency support. Obviously, higher droop actions would result in a power setpoint $P_{i}^{*}$ that exceeds the maximum obtainable power $P_{m p p t}$ and would be limited by the power controller of the wind turbine (cfr. Figure 2). The normally symmetric support would be capped in that case and energy losses would increase.

The procedure to construct this Pareto front is repeated for varying periods of the load, i.e. $T_{L}=25,50,100,200$ and $300 \mathrm{sec}$. Not surprisingly, these Pareto fronts coincide (cfr. Figure 5a) because the amplitude of load variations $\Delta L$ determines the reserve power required. Obviously, a higher amplitude increases the energy loss to achieve the same frequency variability $\sigma_{*}$, which is illustrated in Figure 5b.

In conclusion, current control practice does not make efficient use of the kinetic energy in turbines. Smoothing is indeed only achieved by altering the power set-point after curtailing wind power.

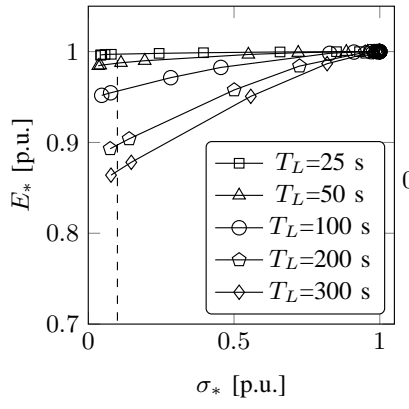

(a)

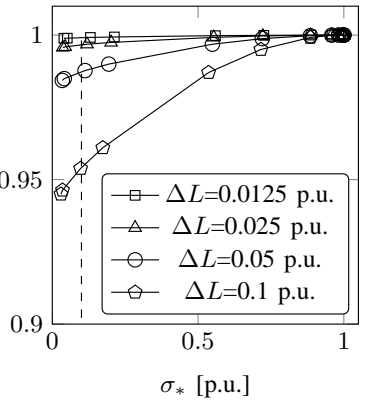

(b)
Fig. 6: Pareto fronts from optimal control simulations (a) where the load period $T_{L}$ is varied with $\Delta L=5 \%$, and (b) where the amplitude $\Delta L$ is varied with $T_{L}=50 \mathrm{sec}$.

\section{B. Optimal system frequency smoothing}

Despite the fact that current controllers do not significantly make use of the kinetic energy reserves in turbines, there is substantial potential for using these reserves at an acceptable energy loss. Optimal control of a wind farm is simulated, with the optimization set-up earlier introduced in $\S$ III to demonstrate the maximum possible benefit that is physically available. The obtained Pareto front shown in Figure 6 clearly shows a dependency of the trade-off both on $\Delta L$ and $T_{L}$.

Starting from $\left(\sigma_{*}, E_{*}\right)=(1,1)$, the Pareto points shift toward lower $\sigma_{*}$ by lowering weight $\alpha$ in Eq. (12). Contrary to causal time-domain results, these Pareto fronts have a quasi horizontal course starting from $\left(\sigma_{*}, E_{*}\right)=(1,1)$, indicating efficient use of kinetic energy. Up to periods of $T_{L}=50 \mathrm{sec}$ and amplitudes of $\Delta L=0.05$ p.u., grid variability is reduced below $\sigma_{*}=0.1$ p.u. at an energy loss within $2 \%$. Smoothing longer periods or larger amplitudes comes at higher energy losses to reduce grid frequency variations. These larger load imbalances have an energy content that exceeds an acceptable exchange of kinetic energy. Such large exchanges of kinetic energy require rotational speeds that push the rotor far away from maximum efficiency. Such behavior is illustrated by plotting the farm power $P_{f}$ :

$$
P_{f}=P_{a, f}+P_{k}, \quad \text { with } \quad P_{a, f}=\sum_{i=1}^{N} P_{a, i},
$$

and where $P_{a, f}$ is the aerodynamic farm power and $P_{k}$ the power extracted for a kinetic energy exchange with the power system. Figure 7 shows both $P_{f}$ and $P_{a, f}$ for $T_{L}=50$ and $100 \mathrm{sec}$ at $\sigma_{*} \approx 0.1$ p.u. (corresponding to the dashed line in Figure 6). Operation around maximum power means that both charges and discharges of the rotor are deviations from maximum efficiency. Thus for optimal operation the ripple on $P_{a, f}$ has half the period of the load variation. The amount of kinetic energy that is exchanged between the power system and the turbine $\Delta E_{k}$, is defined as:

$$
\Delta E_{k}=\int_{t_{c}}^{t_{c}+T_{L} / 2}\left(P_{f}(t)-P_{a, f}(t)\right) \mathrm{d} t,
$$




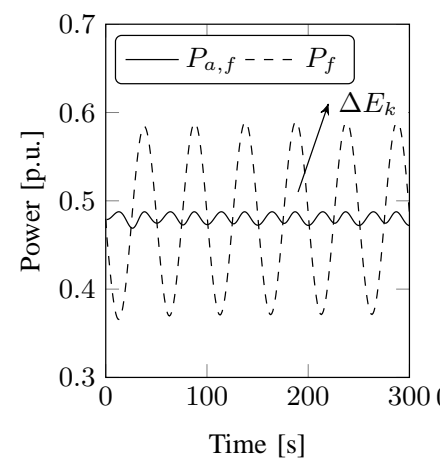

(a) $T_{L}=50 \mathrm{sec}$

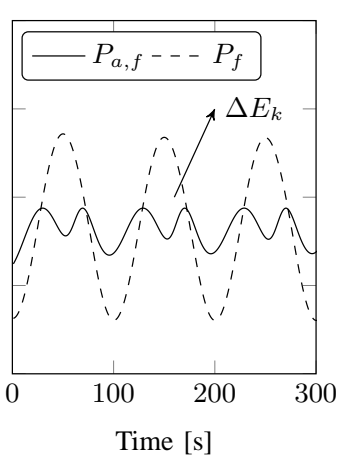

(b) $T_{L}=100 \mathrm{sec}$

Fig. 7: Optimal electric $P_{f}$ and aerodynamic $P_{a, f}$ power profile of two turbines in a power system fed by a variable load. Frequency variations are reduced to $\sigma_{*}=0.1$. p.u.

with $t_{c}$ a randomly chosen time stamp where $P_{f}$ and $P_{a, f}$ cross. This energy exchange clearly rises with period $T_{L}$, or $\left[\Delta E_{k}\right]^{T_{L}=50}<\left[\Delta E_{k}\right]^{T_{L}=100}$. These kinetic energy exchanges are further expressed with respect to the rotating kinetic energy available in turbines when tracking maximum power, obtaining

$$
\kappa=\frac{\left[\Delta E_{k}\right]^{\sigma_{*}=0.1}}{\sum_{i=1}^{n} \frac{I \omega_{m p p t}\left(v_{i}\right)^{2}}{2}} .
$$

For $T_{L}=25,50,100,200,300 \mathrm{sec}$, respectively $\kappa \approx 25$, 50, 90, 180, 270\% ${ }^{1}$. Apparently, larger periods (or larger amplitudes) push the use of kinetic energy to the limit, causing larger deviations of $P_{a, f}$ from the maximum and consequently a lower energy yield, from $E_{*} \approx 0.99$ p.u. $\left(T_{L}=50 \mathrm{~s}\right)$ to $E_{*} \approx 0.96$ p.u. $\left(T_{L}=100 \mathrm{~s}\right)$ for $\sigma_{*}=0.1$ p.u.

Naturally, one would assume that for a limit on the energy loss, also the kinetic energy exchange is limited. This assumption is further explored in Figure 8. This graph depicts the maximum energy yield when increasing the load period and smoothing frequency variability to $\sigma_{*}=0.1$ p.u. Obviously, a higher period embraces larger load variations and accordingly a lower energy yield. The intersection with a limit on the energy loss (e.g. $2 \%$ in Figure 8 ) gives the largest load imbalances (in terms of energy) that can be smoothed at that energy loss. Such load imbalances have an energy content proportional to $T_{L} \Delta L$, which corresponds for every curve with ca. $\kappa \approx 50 \%$. In conclusion, we observe that a kinetic energy exchange of $50 \%$ is feasible when the energy loss is limited to $2 \%$. Of course, this number corresponds to the operating points of turbines and is subject to wind speed changes. Nevertheless, this measure clearly indicates that the energy content of load imbalances determines the ability to smooth grid variations.

\section{Standard control versus optimal system frequency smoothing}

We compare the ability of wind farms to smooth system frequency fluctuations from a standard controller in timedomain simulation with optimal control simulations. To this

\footnotetext{
${ }^{1}$ The latter value that corresponds to $T_{L}=300 \mathrm{sec}$ is far beyond acceptable rotor speed limits and is only added for conceptual purposes.
}

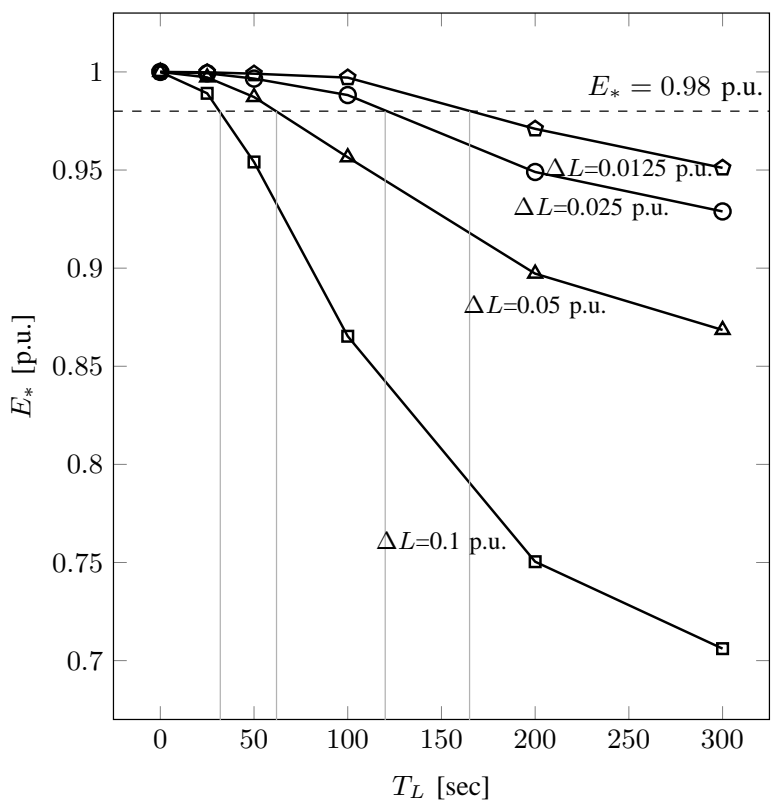

Fig. 8: Maximum energy yield after smoothing frequency variations $\left(\sigma_{*}=0.1\right.$ p.u.), when subject to load variations with amplitude $\Delta L$ and period $T_{L}$.

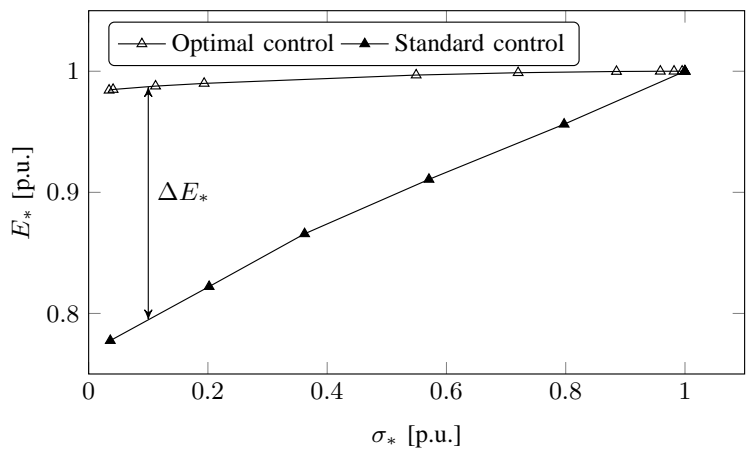

Fig. 9: Pareto front comparison for optimal control and a standard controller facing load variations with period $T_{L}=50 \mathrm{sec}$.

end, Pareto fronts from both approaches are illustrated for a load period of $T_{L}=50 \mathrm{sec}$ in Figure 9. For a standard frequency variation, the benefit in energy yield is clearly distinguishable, e.g. for $\sigma_{*}=0.1$ p.u., $\Delta E_{*}=0.19$ p.u. This gain is due to an improvement of the control strategy, but also perfect knowledge of the incoming wind and load, and coordination of the individual turbines add to $\Delta E_{*}$. The authors do not claim that the chosen standard controller is best to smooth system frequency fluctuations, but only aim at showing the difference in performance with an optimal benchmark.

\section{EFFECT OF INCREASING WIND SHARES}

In addition to the theoretical case introduced above, a load profile that contains a representative frequency spectrum is analyzed to validate earlier observations. To this end, a frequency 
measurement of the West-European grid on a weekday evening is used, between 19:30 and 20:30. The measured frequency profile is converted to a load profile. The frequency profile $\Delta \omega_{s}(t)$ serves as input to the differential Eqs. (2-3) to calculate the governing action $\Delta P_{g}$. This generation profile allows to calculate the load profile $\Delta P_{L}$ by solving Eq. (1) after discretization, and assuming that $\Delta P_{f}=0$. Consequently, this load profile comprises all sources of variability that add to the system frequency variations.

Moreover, the value of the kinetic energy in a power system is analyzed with a much higher wind share, i.e. 90\%. It is expected that wind power, and more generally convertercoupled power sources, will replace conventional synchronous generators to a large extent. Especially during low load and high wind in-feed, such a scenario is plausible. As a consequence, grid-connected inertia and conventional governing action is expected to reduce. Therefore, the value of kinetic energy in wind farms is evaluated in such circumstances.

\section{A. Optimal system frequency smoothing}

The trade-off between maximum energy yield and minimum system frequency variations is again illustrated by means of a Pareto front. For these simulations, the potential is addressed solely by using the optimization analysis.

When underlaying data involve different wind shares $\mu$, the comparison of Pareto fronts is achieved by a normalization with respect to a common reference, thus

$$
\begin{aligned}
& E_{+}=\frac{E}{[E]^{\mu=1 / 5, \alpha=1}} \\
& \sigma_{+}=\frac{\sigma}{[\sigma]^{\mu=1 / 5, \alpha=1}}
\end{aligned}
$$

A first analysis covers the Pareto front with a wind share of $20 \%$, i.e. $\mu=1 / 5$, depicted in Figure 10. The shape of this front corresponds to earlier results. Three simulations runs on this front, respectively Case $A, B$, and $C$, are further investigated in time and frequency domain, respectively illustrated by Figures 11a and 11b. Note that the original frequency profile (Case $A$ ) clearly shows a large swing halfway the simulation, representing power imbalances centered around the hour, caused by hourly market actions.

The first smoothing effort reduces frequency variations down to $10 \mathrm{mHz}$ (ca. $1 \mathrm{~min}$ ), illustrated by the density spectrum for Case $B$ (conceptually represented in Figure 12). This effort comes at an energy loss of less than $2 \%$. Further reductions require a significant energy loss, e.g. above $8 \%$ for Case $C$. At such an energy loss, the system frequency is smoothed down to much lower frequencies, reaching $1 \mathrm{mHz}$. However, rotor speeds exceed the normal operating range, reaching ca. $40 \%$ and $25 \%$ above respectively rated $\left(\omega_{N}\right)$ and maximum rotor speed of a representative variable speed wind turbine. Note that for a rotor speed increase of $40 \%$ above $\omega_{N}$, the kinetic energy nearly doubles. Although simulations runs like Case $C$ are added for conceptual purposes, they show the huge amount of rotating kinetic energy for frequency regulation. Alternatively, pitching allows to smooth these lower

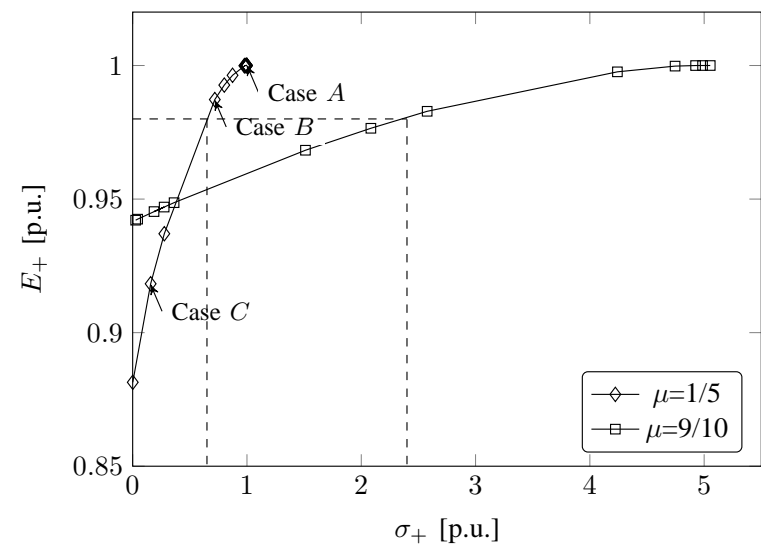

Fig. 10: Pareto fronts for $1 \mathrm{~h}$ optimization runs with a representative load profile and a wind share of respectively $20 \%$ and $90 \%(\mu=1 / 5$ and $9 / 10)$.

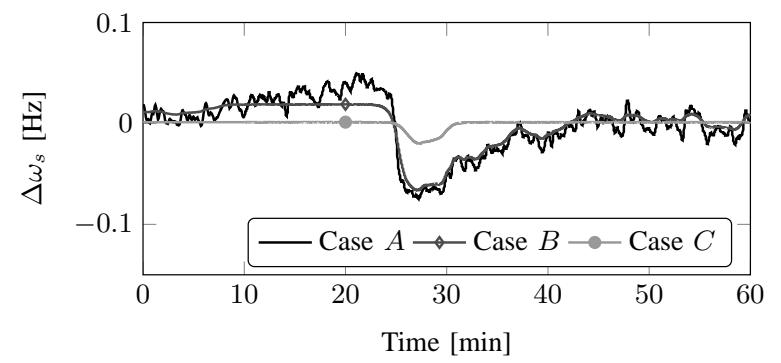

(a)

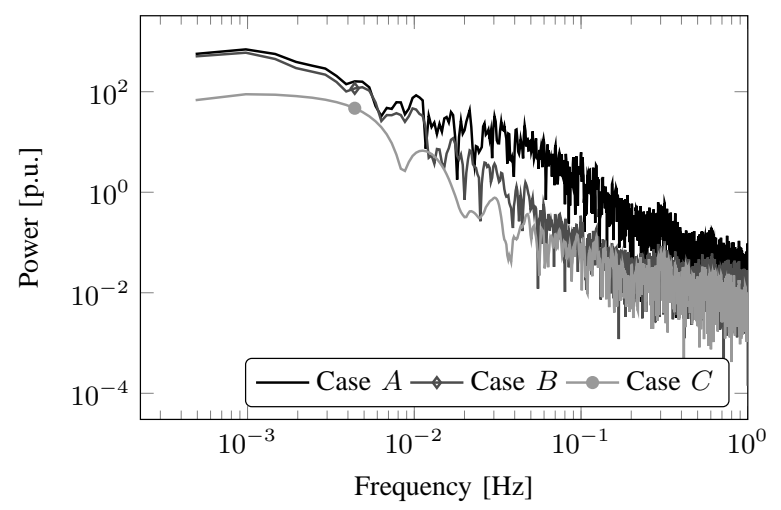

(b)

Fig. 11: (a) Time series and (b) power density spectrum of the system frequency for a wind share of $20 \%$. The Cases correspond to Figure 10.

frequency variations without exceeding rotor speed limits, of course at a higher energy loss.

An increasing wind share up to $90 \%$ raises system frequency variability. The point $\left[\left(\sigma_{+}, E_{+}\right)\right]^{\alpha=1}$ shifts to the right by a factor of ca. 5 in Figure 10. This increased variability is the result of a reduced system inertia $H$ and governing action $1 / R_{g}$, which decrease proportionally with the online capacity of conventional generation. However, variability is reduced at 


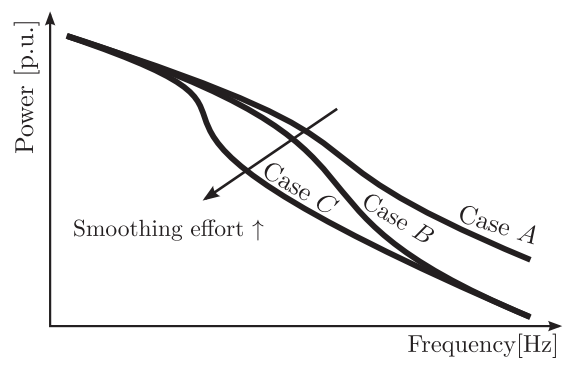

Fig. 12: Conceptual representation of the impact of smoothing on the power density spectrum of system frequency variations.

a lower energy loss; an energy loss of $2 \%$ reduces $\sigma_{+}$with $50 \%\left(\sigma_{+} \approx 2.5\right.$ p.u.) for a wind share of $90 \%$, while only a reduction of $35 \%$ is achieved for a wind share of $20 \%$ (cfr. Figure 10). This observation demonstrates the potential use of kinetic energy from wind farms at an acceptable energy loss, especially with higher instantaneous shares of wind power.

\section{CONCLusions}

We found that the potential of kinetic energy to smooth frequency variations is related to the energy content of accompanied power variations: smoothing variations with an energy content of around $50 \%$ of the kinetic energy comes at an energy loss of $2 \%$. For a wind share of $20 \%$ in the instantaneous generation mix, this potential leads to smoothing frequency variations down to $10 \mathrm{mHz}$, i.e. the minute-tominute time scale. Moreover, this potential increases with the share of wind in the generation mix, possibly smoothing slower frequency variations that correspond to even larger energy contents. In conclusion, kinetic energy in wind turbines has the ability to contribute to frequency regulation within a power system.

Contrary to optimal control, standard controllers for frequency support by wind turbines make use of a power reserve margin. As a consequence, they face an energy loss that is much higher for the same performance, ca. $19 \%$ for the controller here employed. This increased loss originates from three benefits of optimal control over existing controllers: (i) a perfect forecast of the system frequency, (ii) optimal steering of the kinetic energy in wind farms to counteract changes in the frequency and (iii) coordination of kinetic energy exchange among wind farms (or turbines) that face a different wind speed. The comparison with optimal control also illustrates the maximum gain existing controllers can achieve, and it points out tracks for future research concerning the development of real controllers.

\section{ACKNOWLEDGEMENTS}

S. De Rijcke and J. Driesen acknowledge financial support from the Research Foundation - Flanders (FWO, grant no. G.0376.12) and IDO.
TABLE I: Details of generation units

\begin{tabular}{cccccc}
\hline $\begin{array}{c}\text { Conventional } \\
\text { units }\end{array}$ & $S_{g}[\mathrm{MVA}]$ & $I_{s}\left[\mathrm{kgm}^{2}\right]$ & $1 / R_{g}$ [p.u. $]$ & $\tau_{g}[s]$ & $\tau_{t}[s]$ \\
\cline { 2 - 6 } & 10 & $6 \cdot 10^{2}$ & 20 & 0.2 & 0.3 \\
\hline $\begin{array}{c}\text { Wind } \\
\text { turbine units }\end{array}$ & $S_{w}[\mathrm{MVA}]$ & $I\left[\mathrm{kgm}^{2}\right]$ & $1 / R_{w}$ [p.u. $]$ & $R[m]$ & \\
\cline { 2 - 6 } & 2.3 & $6 \cdot 10^{6}$ & $0-11^{*}$ & 38 & \\
\hline
\end{tabular}

* The droop in the wind turbine controller is changed throughout the simulations

\section{APPENDIX I: GENERATION DATA \\ REFERENCES}

[1] B. Kirby, "Frequency regulation basics and trends," tech. rep., Oak Ridge National Laboratory, 2004.

[2] M. Milligan, P. Donohoo, D. Lew, E. Ela, B. Kirby, H. Holttinen, E. Lannoye, D. Flynn, M. O'Malley, and N. Miller, "Operating reserves and wind power integration: an international comparison," in proc. 9th International Workshop on large-scale integration of wind power into power systems, pp. 18-29, 2010.

[3] EURELECTRIC and ENTSO-E, "Deterministic frequency deviations root causes and proposals for potential solutions," tech. rep., EURELECTRIC and ENTSO-E, December 2011.

[4] H. Banakar, C. Luo, and B. T. Ooi, "Impacts of wind power minuteto-minute variations on power system operation," IEEE Transactions on Power Systems, vol. 23, no. 1, pp. 150-160, 2008.

[5] H. Holttinen and R. Hirvonen, Power System Requirements for Wind Power, ch. 8, pp. 143-167. John Wiley \& Sons, Ltd, 2005.

[6] B. G. Rawn and P. W. Lehn, "Wind rotor inertia and variable efficiency: Fundamental limits on their exploitation for inertial response and power system damping," in Proc. Eur. Wind Energy Conf., 2008.

[7] B. G. Rawn, M. Gibescu, and W. L. Kling, "Kinetic energy from distributed wind farms: Technical potential and implications," in Innovative Smart Grid Technologies Conference Europe (ISGT Europe), 2010 IEEE PES, pp. 1-8, IEEE, 2010.

[8] L. Ruttledge and D. Flynn, "Co-ordination of frequency responsive wind plant in future power systems," in Renewable Power Generation Conference (RPG 2013), 2nd IET, pp. 1-4, IET, 2013.

[9] R. De Almeida and J. Peças Lopes, "Participation of doubly fed induction wind generators in system frequency regulation," IEEE Transactions on Power Systems, vol. 22, no. 3, pp. 944-950, 2007.

[10] G. Ramtharan, J. Ekanayake, and N. Jenkins, "Frequency support from doubly fed induction generator wind turbines," IET Renewable Power Generation, vol. 1, no. 1, pp. 3-9, 2007.

[11] J. Morren, S. W. H. De Haan, W. Kling, and J. Ferreira, "Wind turbines emulating inertia and supporting primary frequency control," IEEE Transactions on Power Systems, vol. 21, no. 1, pp. 433-434, 2006

[12] G. Lalor, A. Mullane, and M. O’Malley, "Frequency control and wind turbine technologies," IEEE Transactions on Power Systems, vol. 20, no. 4, pp. 1905-1913, 2005.

[13] European Network of Transmission System Operators for Electricity (ENTSO-e), "Supporting document for the network code on loadfrequency control and reserves," tech. rep., ENTSO-e, 2013. 156 pages.

[14] S. De Rijcke, Dynamic grid support by wind farms: potential of rotating kinetic energy. PhD thesis, KU Leuven, 2014.

[15] B. G. Rawn, P. W. Lehn, and M. Maggiore, "Disturbance margin for quantifying limits on power smoothing by wind turbines," Control Systems Technology, IEEE Transactions on, vol. 21, no. 5, pp. 1795$1807,2013$.

[16] W. Li, G. Joos, and C. Abbey, "Wind power impact on system frequency deviation and an ess based power filtering algorithm solution," in Power Systems Conference and Exposition, 2006. PSCE'06. 2006 IEEE PES, pp. 2077-2084, IEEE, 2006.

[17] M. Wang-Hansen, R. Josefsson, and H. Mehmedovic, "Frequency controlling wind power modeling of control strategies," IEEE Transactions on Sustainable Energy, vol. 4, no. 4, pp. 954-959, 2013.

[18] L.-R. Chang-Chien, W.-T. Lin, and Y.-C. Yin, "Enhancing frequency response control by dfigs in the high wind penetrated power systems,", IEEE Transactions on Power Systems, vol. 26, no. 2, pp. 710-718, 2011.

[19] P. Kundur, Power system stability and control. McGraw-Hill, Inc., 1994.

[20] N. Miller, J. J. Sanchez-Gasca, W. Price, and R. Delmerico, "Dynamic modeling of GE 1.5 and 3.6 MW wind turbine-generators for stability simulations," in IEEE Power Engineering Society General Meeting, vol. 3, pp. 1977-1983 Vol. 3, 2003. 
[21] A. Hansen, P. Sørensen, F. Iov, and F. Blaabjerg, "Centralised power control of wind farm with doubly-fed induction generators," Renewable Energy, vol. 31, pp. 935-951, 2006.

[22] J. G. Slootweg, H. Polinder, and W. L. Kling, "Dynamic modelling of a wind turbine with doubly-fed induction generator," in Vancouver IEEE Power Engineering Society Summer Meeting, 15-19 July 2001.

[23] S. Heier, Grid integration of wind energy conversion systems. Wiley, 2005.

[24] P. Rutquist and M. Edvall, User's Manual for TOMLAB. 1260 SE Bishop Blvd Ste E, Pullman, WA 99163, USA: Tomlab Optimization Inc., November 2008

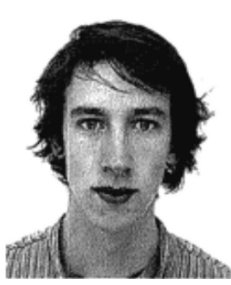

Simon De Rijcke (S'08) was born in Belgium, on May 26, 1986. He graduated as an electrotechnical engineer from the KU Leuven in 2009 and received a master of science in energy. He finished his $\mathrm{PhD}$ on dynamic grid support by wind farms in 2014 in the research group ELECTA, department of Electrical Engineering of the KU Leuven. He started working for Nordex Energy GmbH as a development engineer in August 2014. His research interests are power system control with the integration of renewable energy sources, wind turbine and farm control.

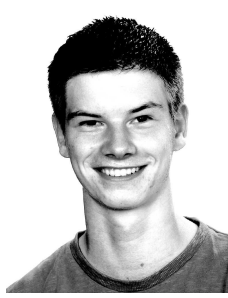

Pieter Tielens (S'11) was born in 1988, in Genk, Belgium. He received the M.Sc. degree in electrical energy engineering from the KU Leuven, Belgium, in 2011, where he is currently working towards a $\mathrm{Ph} . \mathrm{D}$. degree. He is a Research Assistant in the research group Electa, department of Electrical Engineering of the KU Leuven. His fields of interest are the integration of renewable energy sources into the electricity system and power systems with low inertia.

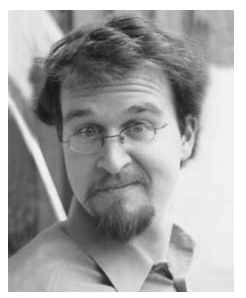

Barry Rawn (M'10) received the Ph.D. degree in electrical engineering in 2010 from the University of Toronto, Canada, where he also received the B.A.Sc. and M.A.Sc. degrees in engineering science and electrical engineering.

His research interests include nonlinear dynamics and sustainable energy infrastructure. He is currently a postdoctoral researcher in the Electa group at the KU Leuven, Belgium.

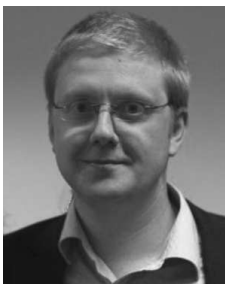

Dirk Van Hertem (S'02, SM'09) was born in 1979, in Neerpelt, Belgium. He graduated as a M.Eng. in 2001 from the KHK, Geel, Belgium and as a M.Sc. in Electrical Engineering from the KU Leuven, Belgium in 2003. In 2009, he has obtained his $\mathrm{PhD}$, also from the KU Leuven.

In 2010, Dirk Van Hertem was a member of EPS group at the Royal Institute of Technology, in Stockholm, Sweden where he was the program manager for controllable power systems for the $\mathrm{EKC}^{2}$ competence center at KTH. Since spring 2011 he is back at the University of Leuven where he is an assistant professor in the ELECTA group. His special fields of interest are power system operation and control in systems with FACTS and HVDC and building the transmission system of the future, including offshore grids and the supergrid concept. $\mathrm{He}$ is an active member of both IEEE (PES and IAS) and Cigré.

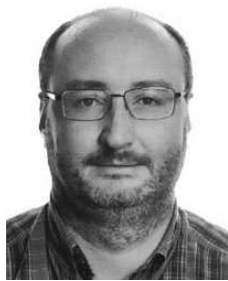

Johan Driesen (S'93M'97SM'12) received the M.Sc. and $\mathrm{Ph} . \mathrm{D}$. degrees in electrical engineering from the KU Leuven, Belgium, in 1996 and 2000, respectively.

Currently, he is a Professor with the KU Leuven and teaches power electronics and electric drives. In 2000, he was with the Imperial College of Science, Technology and Medicine, London, U.K. In 2002, he was with the University of California, Berkeley. Currently, he conducts research on distributed generation, power electronics, and its applications. 